\title{
Motion Simulation and Dynamic Analysis of a Four- Cylinder Wobble-Plate Compressor
}

\author{
Yong-guang Liu \\ College of automation science and electrical engineering \\ Beihang University \\ Beijing, China, 13681055187 \\ lyg@buaa.edu.cn
}

\begin{abstract}
In this paper we provide a detailed analysis on a fourcylinder wobble-plate compressor in the field of the motion simulation and dynamics. Then, according the results of the analysis, we program with MATLAB to come to the motion curve of the pistons, as well as the curves on the gas force, the piston force, and the resistance moment, which will make us analysis the changes of the motion and force easily.
\end{abstract}

Keywords-wobble plate compressor; motion simulation; dynamics.

\section{INTRODUCTION}

Swash plate compressor is widely used in automotive air conditioning systems. It has two structures: the swash plate and the wobble plate. Light weight, small size, compact structure, put the wobble plate compressor widely used. In this article we provide a detailed analysis on the wobble plate compressor in the field of the motion simulation and dynamic, which will give us a fully understand on movement variation of each piston and the force changes of compressors. Then, it will be a reference for similar models.

\section{WORKING PRINCIPLE}

A simple model of the wobble plate compressor is shown in Figure 1. The basic elements of the compression mechanism are the shaft-drive plate assembly, the sliding shaft sleeve, the rotating journal and the wobble plate. With the drive plate fixed to the drive shaft, the wobble plate close on the inclined plane of the drive plate, forming a certain angle. The wobble plate is connected to each of the four pistons with a double ended ball connected rod. The wobble plate translates the journal motion into linear reciprocation of the pistons ${ }^{[1]}$.

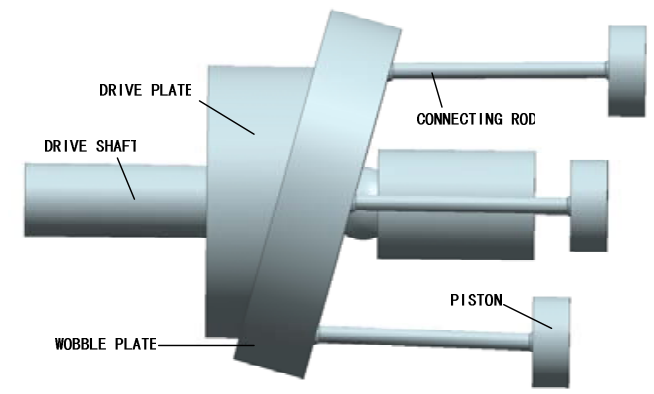

Figure 1. Schematic diagram of the wobble plate compressor.

\author{
Guo Hui \\ College of automation science and electrical engineering \\ Beihang University \\ Beijing, China, 15210800795 \\ guooohui@163.com
}

\section{MOTION SIMULATION}

The motion simulation is the main part of UG/Motion module, which will help us make the complex kinematic analysis, dynamic analysis and design simulation for any twodimensional or three-dimensional structure. The rationality of $t$ of the structure designed can be verified by the kinematics or dynamic motion analysis. We can take advantage of the various parts of the graphics output displacement, acceleration, velocity and force changes. Motion simulation curves of the four pistons are shown in Figure 2.

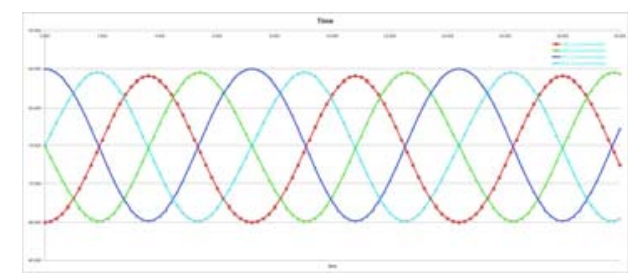

Figure 2. Motion simulation curves of the four pistons.

The horizontal axis represents the time, and the vertical axis represents the displacement. It can be seen from the figure, the pistons move in a cosine curve and in the same trip when the drive shaft is rotating, and they are followed by a difference of $0.5 \pi$ phase angle.

\section{DYNAMIC ANALYSIS}

\section{A. Piston force}

The piston force includes gas force, the reciprocating inertia force and the friction of the piston and cylinder wall. Gravity is negligible in the analysis.

\section{1) Gas force}

Gas force acting on the piston comes from the cylinder and the back side of the piston. The former varies with the rotation corner $\theta$ of the drive shaft, the latter can be approximately considered constant. Thus the gas force should be the product of gas pressure on both sides of the piston and the area, and we should define the direction of the piston compression as a positive direction, so

$$
F_{g}=\left(p-p_{s}\right) A_{p}
$$


$p$ is the gas pressure within the cylinder, $p_{s}$ is the inlet pressure, $A_{p}$ is the area of the piston.

As shown in Figure 3, one compression cycle is divided into four processes. If we define the maximum displacement of the piston as a starting point, four processes are as follows: expansion process, intake process, compression process and exhaust process. In the expansion process and compression process, the gas is based on isothermal change, and the gas is about to leave the pressure unchanged in the intake $\mathrm{s}$ and exhaust process. The specific process is as follows ${ }^{[2]}$ :

Expansion process $(1 \rightarrow 2)$ :

$$
p=\left(\frac{V_{1}}{V}\right)^{m} p_{1}=\left[\frac{a_{c} S}{a_{c} S+R_{p} \tan \alpha(1-\cos \theta)}\right]^{m} p_{1}
$$

Intake process $(2 \rightarrow 3)$ :

$p=p_{2}$, the pressure will not be changed until that $\theta=\pi$.

Compression process $(3 \rightarrow 4)$ :

$$
p=\left(\frac{V_{3}}{V}\right)^{m} p_{3}=\left[\frac{a_{c} S+S}{a_{c} S+R_{p} \tan \alpha(1-\cos \theta)}\right]^{n} p_{2}
$$

Exhaust process $(4 \rightarrow 1)$ :

$p=p_{1}$, the pressure will not be changed until that $\theta=2 \pi$.

$\mathrm{S}$ is the piston stroke, $\mathrm{a}_{\mathrm{c}}$ is the relative clearance volume, $\mathrm{R}_{\mathrm{p}}$ is the radius of the cylinder distributed, $\mathrm{p}_{1}$ is the actual discharge pressure, $\mathrm{p}_{2}$ is the actual inlet pressure.

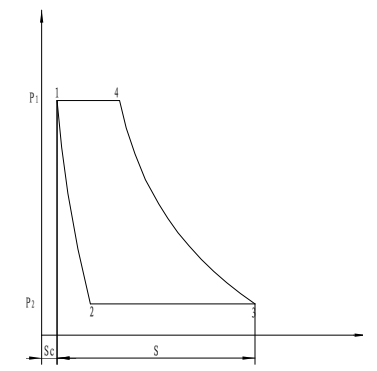

Figure 3. One compression cycle.

According to the formula of the cyclical process, we program with MATLAB to come to the motion curves. The gas pressure changes of four cylinders are shown in Figure 4. The gas forces of the pistons are shown in Figure 5. Take the change of gas force of the cylinder 1 as an example, the piston begin to swell at the maximum displacement, at the same time, the gas force is gradually reduced. To a certain point, the gas force is zero, then, the cylinder begins to intake until the piston reaches the minimum displacement $(\pi)$, at this point, the end of the intake. Then the piston starts to compress, and the gas force is gradually increasing. When the required pressure is reached, the exhaust valve opens to exhaust. Exhaust process will not come to an end until the piston reaches the maximum displacement $(2 \pi)$.At this time, the cycle is complete. The gas force changes of the other cylinder can be drawn from the motion simulation curve of the piston. When Cylinder 1 is going to expand, cylinder 2 is compressing, and cylinder 3 will to compress, and cylinder 4 is inhaling.

\section{2) Reciprocating inertia force}

We often call the reciprocating moving parts the piston group. Expression of the reciprocating inertia force:

$$
I=m a=m R_{q} \omega^{2} \tan \alpha \cos \theta
$$

A is the acceleration of the piston, $\theta$ is the rotating angle of the drive shaft, $\omega$ is the angular velocity of the drive shaft.

The change of the reciprocating inertia force is shown in Figure 6. Take the change of reciprocating inertia force of the piston 1 as an example, when the piston reaches the maximum displacement and minimum displacement, the reciprocating inertia force reaches the maximum. When the drive shaft rotates to $0.5 \pi$ and $1.5 \pi$, the reciprocating inertia force is zero. Define the maximum displacement of the piston 1 as the initial position, the reciprocating inertia forces of the other pistons are shown in Figure 6.

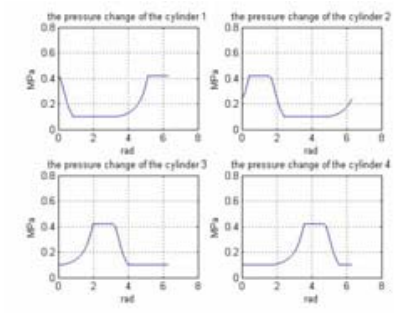

Figure 4. The gas pressure
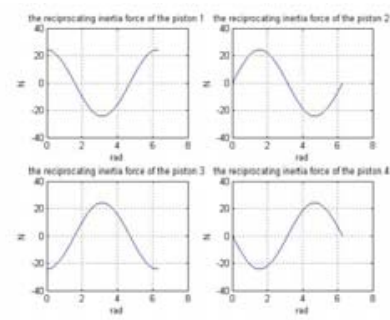

Figure6. The reciprocating inertia force
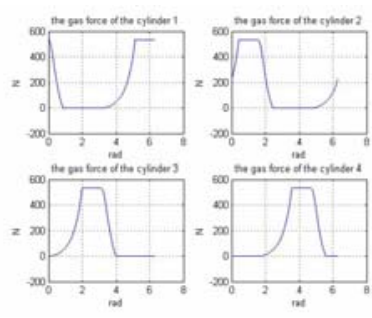

Figure5. The gas force

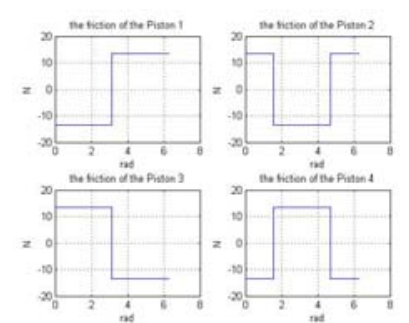

Figure7. The reciprocating friction

\section{3) Reciprocating friction}

We could simplify the reciprocating friction as a constant force. Approximate formula is as follows:

$$
F_{f}=\frac{A_{p}}{16}\left(p_{s}+p_{d}\right)\left(\frac{1}{\eta_{m}}-1\right)
$$

$A_{p}$ is the area of the piston, $p_{s}$ is the inlet pressure of the cylinder, $p_{d}$ is the discharge pressure of the cylinder, $\eta_{m}$ is the mechanical efficiency of the machine.

The changes of the reciprocating friction are shown in Figure 7. Take the change of reciprocating friction of the piston 1 as an example, the friction is constant throughout the cycle. The friction is negative in the expansion process and intake process, and the friction is positive in the compression process and exhaust process. Define the maximum displacement of the 
piston 1 as the initial position, the reciprocating friction of the other pistons are shown in Figure 7.

\section{4) Piston force}

The piston force includes the gas force of the cylinder, the reciprocating inertia force of the piston and the reciprocating friction.

$$
F_{p}=F_{g}+F_{f}+I
$$

The changes of the piston force are shown in Figure 8. Take the change of the piston force of the piston 1 as an example, the horizontal axis represents the radian, and the vertical axis represents the force. The piston force will gradually reduce in the expansion process. It will begin to inhale when the drive shaft rotates $0.873 \mathrm{rad}$, and will stop inhaling when the drive shaft rotates $\pi \mathrm{rad}$. Then the piston starts to compress, the friction gets into reverse and the curve is going to jump. The exhaust valve will not open until the drive shaft rotates 5.136 rad. Then it begins to exhaust until the drive shaft rotates $2 \pi$. The piston forces of the other pistons are shown in Figure 8.
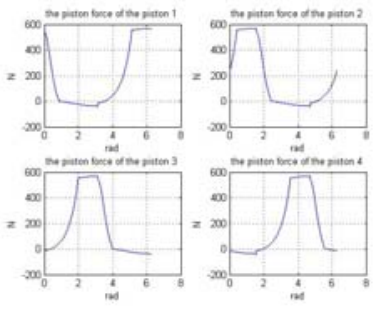

Figure8. The piston force
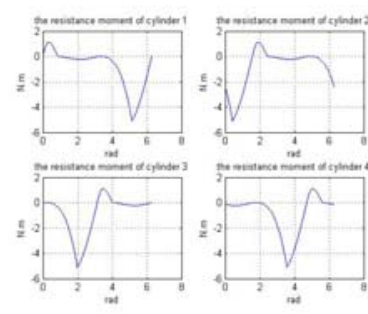

Figure10. The resistance moment

\section{B. Total resistance moment}

The total resistance moment of the compressor is equal to the total frictional resistance moment and the resistance moment of all the cylinders ${ }^{[3]}$.

$$
M=\sum_{i=1}^{4} M_{i}+M_{t}
$$

1) Resistance moment of the cylinders

The stress analysis of the wobble plate compressor is shown in Figure 9.
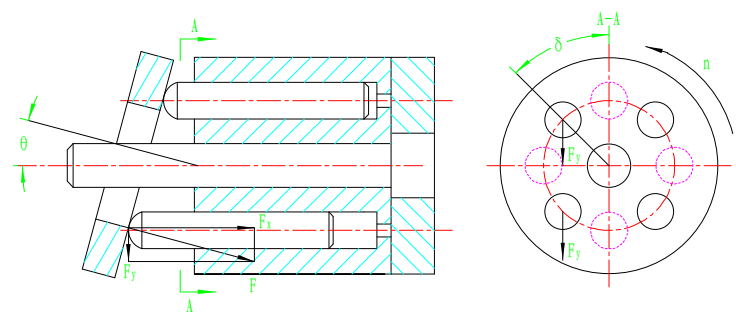

Figure9.Stress condition of the wobble plate compressor

Set the plunger diameter of the compressor as $d$ and set the input pressure as $p$, by the force equilibrium conditions can be obtained. Expression can be obtained by the equilibrium condition of the force:

$$
\begin{gathered}
F_{x}=\frac{\pi}{4} d^{2} \cdot p \\
F_{y}=F_{x} \cdot \tan \theta=\frac{\pi}{4} d^{2} \cdot p \cdot \tan \theta
\end{gathered}
$$

Set the radius of the cylinder distributed as $D_{p}$ and set the angle between the location of a plunger and the center line of the cylinder as $\theta$. Instantaneous torque generated by the plunger:

$$
T=F_{y} \cdot D_{p} \cdot \sin \delta=\frac{\pi}{4} \cdot d^{2} \cdot p \cdot \tan \theta \cdot D_{p} \cdot \sin \delta
$$

The total instantaneous torque of the wobble plate type compressor should be the sum of the plunger torque:

$$
\begin{gathered}
T_{t}=\sum_{i=1}^{4} M_{i}=\sum\left(\frac{\pi}{4} d^{2} \cdot p \cdot \tan \theta \cdot D_{p} \cdot \sin \delta\right) \\
F_{p}=\frac{\pi}{4} d^{2} \cdot p
\end{gathered}
$$

The change of resistance moment of each cylinder is shown in Figure 10.

2) The frictional resistance moment

The total frictional resistance moment includes the resistance moment between the wobble plate and the bearing of the wobble plate, also includes the resistance moment between the drive plate and the bearing of the drive plate. The resistance moment are shown in Figure 11, Figure 12.

$$
M_{t}=M_{p}+M_{r}
$$

$M_{t}$ is the total frictional resistance moment, $M_{p}$ is the resistance moment of the wobble plate, $M_{r}$ is the resistance moment of the drive plate.

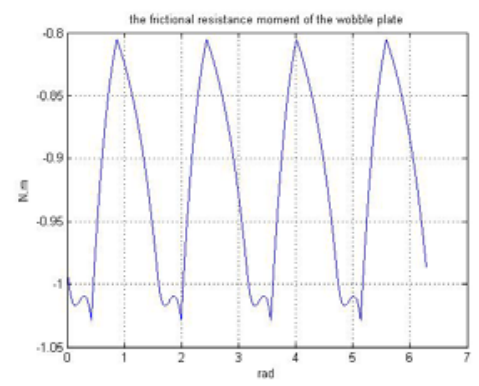

Figure11. Frictional resistance moment

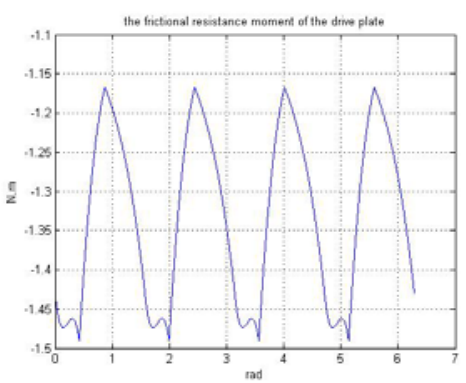

Figure12. Frictional resistance moment 
The frictional resistance moment between the wobble plate and the bearing of the wobble plate:

$$
M_{p}=f_{p} R_{m p}\left(\sum_{i=1}^{4} F_{p i}+F_{s p}\right) / \cos \alpha
$$

$f_{p}$ is the coefficient of friction between the wobble plate and the bearing of the wobble plate, $R_{m p}$ is the average radius of the friction surface between the wobble plate and the bearing of the wobble plate.

The frictional resistance moment between the drive plate and the bearing of the drive plate:

$$
M_{r}=f_{r} R_{m r}\left(\sum_{i=1}^{4} F_{p i}+F_{s p}\right)
$$

$f_{r}$ is the coefficient of friction between the drive plate and the bearing of the drive plate, $R_{m r}$ is the average radius of the friction surface between the drive plate and the bearing of the drive plate.

\section{3) Total resistance moment}

The total resistance moment of the compressor is equal to the total frictional resistance moment and the resistance moment of all the cylinders. The total resistance moment is shown in Figure 13.

$$
M=\sum_{i=1}^{4} M_{i}+M_{t}
$$

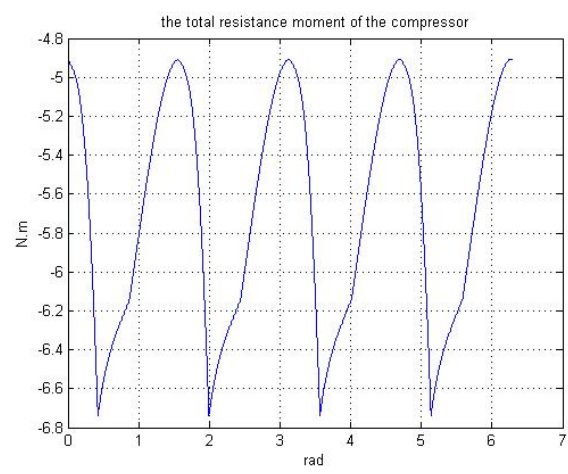

Figure13. The total resistance moment

The size and direction of the piston force is changing all the time because the cylinders are in different position. Some of the piston forces make the connecting rod press the wobble plate, the others make the connecting rod divorce from wobble plate, which makes the work resistance moment of each cylinder changing all the time. The friction resistance moment will change with the drive plate rotating, but only a little change.

\section{CONCLUSION}

After the dynamic analysis and calculation of all the above, the following conclusions can be drawn:

(1)The four pistons of the wobble plate compressor move in a cosine curve and in the same trip when the drive shaft is rotating uniformly, and they are followed by a difference of
$0.5 \pi$ phase angle. This is also the basis for calculating the piston force and the resistance moment. The variation law of the others can be drawn through the piston 1 changes.

(2)The reciprocating inertia force may have been the vibration source of machine. As can be seen from the graph, it is a cosine curve, but the amplitude is small. This type is 4 cylinder structure and the cylinders are distributed uniformly, so that the reciprocating inertia force can almost achieve a balance. So we can make use of the multi-column cylinder structure and the method of trimming the mass of the drive shaft or the swash plate to balance.

(3) The total resistance moment of the wobble plate compressor has been always changing frequently with the drive plate rotating, which is determined by the gas force. That is to say, if the outlet pressure desired is greater, the resistance moment will be greater. The accurate numerical value can be calculated by the outlet pressure designed. Through the variation law of the resistance moment, we can draw the average resistance moment in a rotation period, and we can further get the shaft power of the wobble plate compressor, which will make the drive motor selected easily.

\section{REFERENCES}

[1] Yu Yong-zhang. Positive-displacement compressors technical manuals [M]. Beijing: China Machine Press, 2005.

[2] Yu Yong-zhang. Piston compressor [M]. China Machine Press, 1982.

[3] Institute of Compressor, Xian Jiaotong University. The design of the piston compressor [M]. Xi'an: Xi'an Jiao Tong University press, 2009.

[4] Jin Xiao-xiong, Huang Suo-cheng. The dynamic analysis and calculation of the SE-508 type wobble plate compressor [J]. Compressor Technology, 2002(5):5-9.

[5] Zhang Hua, Ye Li-guo. The conditioning system scheme of the locomotive compressed air and the design of the key parts [J]. Journal of Northern Jiaotong University,2004, 2(1):87-91

[6] Tian CQ, DouCP. A mathematical model of variable displacement wobble plate compressor for automotive air conditioning system. Applied Thermal Engineering, 2004, 24(17-18): 2467-2486

[7] TianCQ, DouCP. Experimental investigation on the steady-state performance and regulating characteristics of variable displacement wobble plate compressor. Journal of Automobile Engineering, 2005, 219 : 271-281

[8] D.Marsden. System Design Reguirements for Infra-red Wetector Cryocoolers, proc.of the 4th.Int. Conf. on Cryocoolers. p229

[9] Kenji Tojo, et al. A Study on the Kinmatics of a Variable Displacement Compressor for Automotive Air Conditioners, Proc. of ICECP,1988.

[10] Skinner T J, Swadner R L. V-5 Automotive variable displacement air conditioning compressor. SAE Congress Paper 850040 